\title{
INVESTIGATION AND APPLICATION OF PNEUMATIC ARTIFICIAL
}

\section{MUSCLES}

\author{
József Sárosi, Gábor Szabó, János Gyeviki \\ Department of Technical and Process Engineering, Faculty of Engineering \\ University of Szeged \\ sarosi@mk.u-szeged.hu
}

\begin{abstract}
The movement of pneumatic artificial muscle (PAM) is soft that similar to the human muscle therefore it can be used as the actuator for rehabilitation devices and prostheses. In this paper a Fluid Muscle manufactured by Festo is tested, and some experimental results, rehabilitation devices and prostheses are shown. Our goal is to construct an intelligent prosthetic arm using PAMs.
\end{abstract}

Keywords: pneumatic artificial muscle; test-bed; experimental results; therapy devices; prosthetic arms

\section{Introduction}

The pneumatic artificial muscle is a pneumatic actuator with a comprehensive history of applications in the biomechanical field. The most promising pneumatic actuator is evidently the McKibben pneumatic muscle actuator. The McKibben muscle was invented in the 1950's by physician Joseph L. McKibben to help the movement of polio patients and to motorize pneumatic arm orthotics. There exists several types of artificial muscles that are based on the use of rubber or some similar elastic materials, such as the McKibben muscle, the Rubbertuator made by Bridgestone company, Air Muscle made by Shadow Robot company, Fluid Muscle made by Festo company, Pleated PAM developed by Vrije University of Brussel, ROMAC (RObotic Muscle ACtuator), Yarlott and Kukolj PAM and some others. There are a lot of advantages of artificial muscles like the high strength, good power-weight ratio, low price, little maintenance needed, great compliance, compactness, inherent safety and usage in rough environ- ments. Disadvantages are connected with the accuracy of control and nonlinearities of pneumatic systems ${ }^{1,2}$.

Pneumatic muscle actuators consist of a rubber bladder enclosed within a helical braid that is clamped on both ends. As the bladder is pressurized, its volume increases and the braid and clamps act to shorten the overall length of the actuator. The general behaviour of PAM with regard to shape, contraction and tensile force when inflated depends on the geometry of the inner elastic part and of the braid at rest and on the materials used (Figure 1). Typical materials used for the membrane construction are latex and silicone rubber, while nylon is normally used in the fibres ${ }^{3}$.

Pneumatic artificial muscles show similarity to biological muscles. The PAMs are one-way acting, we need two ones to generate bidirectional motion: one of them moves the load, the other one will act as a brake to stop the load at its desired position and the muscles have to change function to move the load in the oppo- 


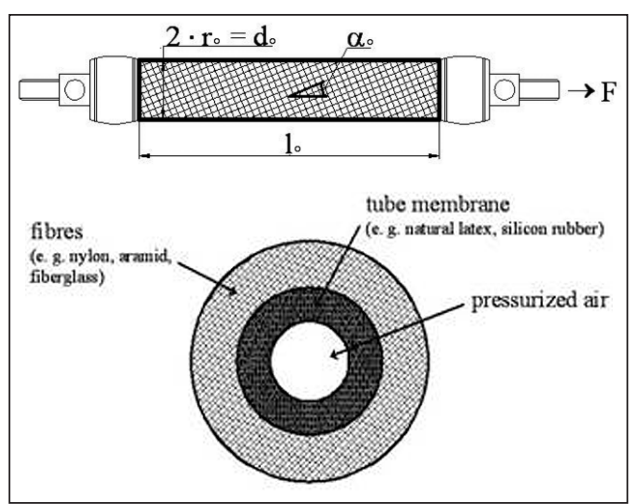

Figure 1. Geometry parameters of PAM and its orthotropic material layers

site direction. This specific connection of the muscles to the load is generally named as an antagonistic set-up $\mathrm{p}^{2,4,5}$.

The Fluid Muscle DMSP-20-200N-RM-RM (with inner diameter of $20 \mathrm{~mm}$ and initial length of $200 \mathrm{~mm}$ ) produced by Festo company is selected for our newest study. Figure 2 shows different Fluid Muscles.

The layout of this paper is as follows. Section 2 (Materials and Methods) is devoted to display our test-bed for investigation of pneumatic muscles. In section 3 (Results and Discussion), we present some experimental results, our new approximation algorithm of force as a function of pressure and length (contraction) and several devices for rehabilitation and prostheses using PAMs. Finally, section 4 (Conclusions and Future Work) gives the future work we plan.

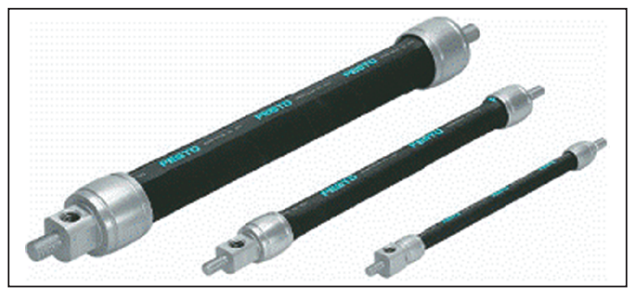

Figure 2. Fluid Muscles made by Festo

\section{Materials and Methods}

Good description of our experimental results can be found in 6 .

The experimental set-up (Figure 3) consists of a slider mechanism. One side of the muscle is fixed to a load cell, while the other side is attached to the movable frame. The load cell (7923 type from MOM) is a 4 bridge element of strain gauges. It is mounted inline to the PAM on the fixed surface. The load cell measures the force exerted by the PAM. The tests are performed by changing the displacement of this slider. The linear displacement of the actuator is measured using a LINIMIK MSA 320 type linear incremental encoder with $0.01 \mathrm{~mm}$ resolution. During each test, frame position, muscle force and applied gauge pressure are recorded.

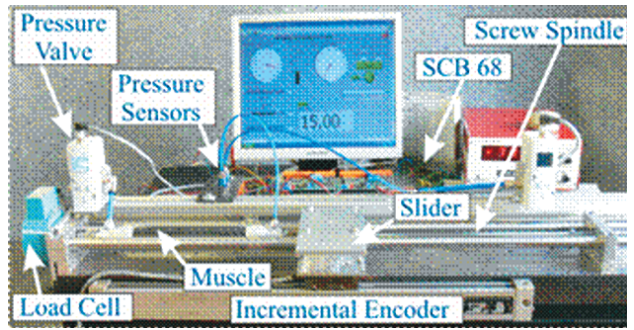

Figure 3. Experimental setup for investigations of PAMs

In the test-bed two fluidic muscles can be mounted. Instead of second PAM a bias spring or an external load can be attached with a flexible steel cable, producing the necessary counter force to pull the actuator back when it is not activated.

The air pressure applied to the actuators can be regulated with two adjustable regulators type Festo VPPM-6L-L-1-G1/8-0L6H-V1N$\mathrm{S} 1 \mathrm{C} 1$. The proportional pressure regulators (PPRs) are controlled by voltage inputs. The main purpose of the PPR is to regulate the 


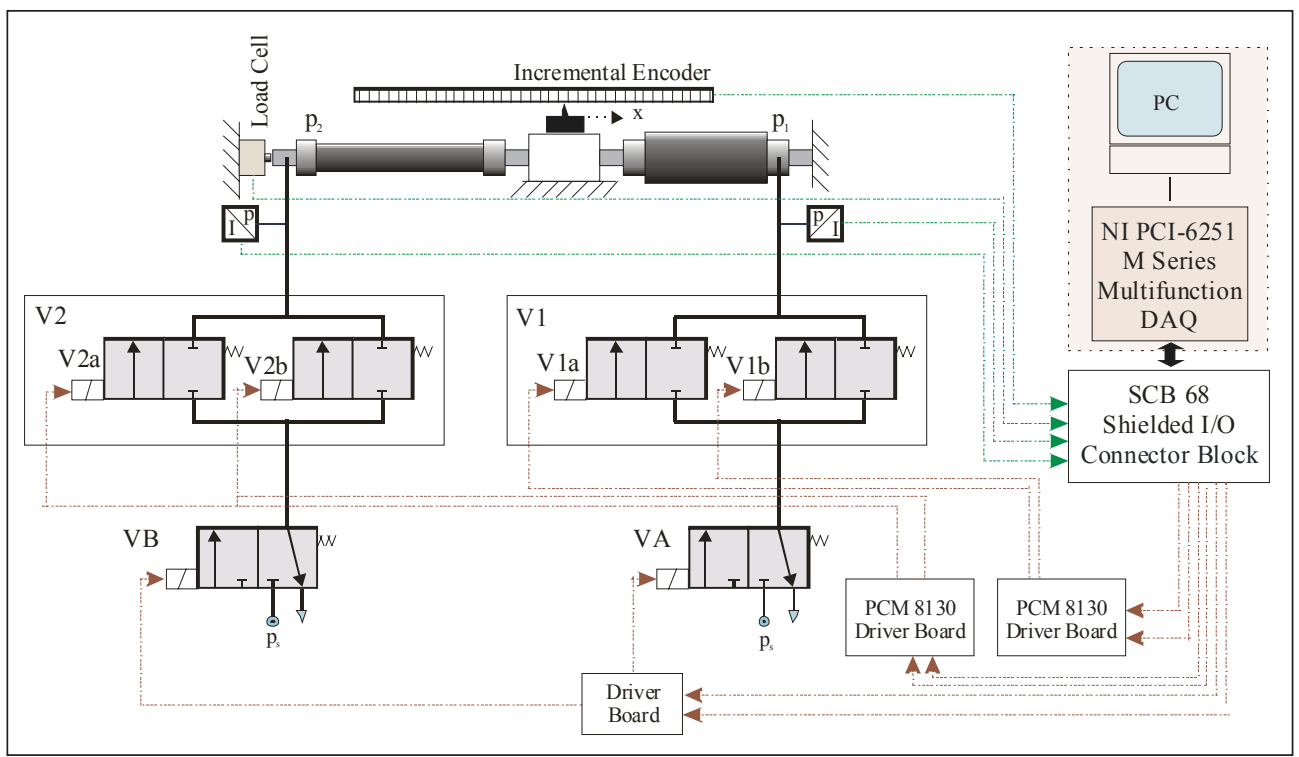

Figure 4. Configuration of pneumatic positioning system with ON/OFF valves

pressure entering the PAM. To measure the air pressure, two Motorola MPX5999D pressure sensors were plumbed into the pneumatic circuit. A National Instruments Multi-I/O card (NI 6251) reads the signal of force, pressure sensors and incremental encoder into the PC.

National Instruments LabVIEW is a typical example for high level software, capable of connecting various kinds of DAQ boards with a PC. We used this program to monitor and collect the data imported through the DAQ card. It also dispatches the control profiles for the PPRs.

For positioning, in the test-bed, two Fluidic Muscles can be controlled by tree-way and twoway solenoid valves (MATRIX HX 751.102 C 324 3/2 NC and PX 861.9E4C2KK fast switching types) (Figure 4).

\section{Results and discussion}

Tensile force of artificial muscle under different constant pressures is a function of muscle length (contraction) and of air pressure. The force always drops from its highest value at full muscle length to zero at full inflation and position (Figure 5).

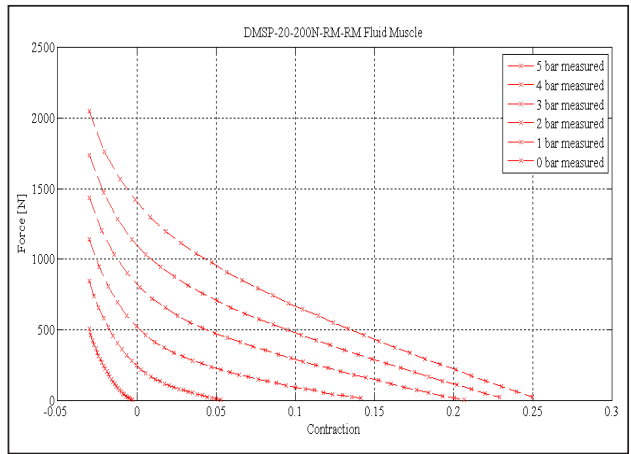

Figure 5. Isobaric force-contraction diagram of Fluid Muscle

Many researchers have investigated the behaviour of PAM and some of them have introduced different mathematical models for this actuator. However, we have noticed significant differences between the theoretical and experimental results. Therefore, we have worked out a better approximation algorithm 
for the equation of force. Under fixed pressure the contraction to force function can be approximated by an exponential function:

$\mathrm{F}(\kappa)=\mathrm{a} \cdot \mathrm{e}^{(\mathrm{b} \cdot \kappa+\mathrm{c})}+\mathrm{d} \cdot \kappa+\mathrm{e}$

To make our equation 1 universal meaning usable under various pressures we need to make the algorithm vary from pressure:

$$
\begin{aligned}
\mathrm{F}(p, \kappa)= & (\mathrm{a} \cdot \mathrm{p}+\mathrm{b}) \cdot \mathrm{e}^{(\mathrm{c} \cdot \kappa+\mathrm{d})}+(\mathrm{e} \cdot \mathrm{p}+\mathrm{f}) \cdot \kappa+ \\
& +\mathrm{g} \cdot \mathrm{p}+\mathrm{h}
\end{aligned}
$$

The unknown $a, b, c, d, e, f, g$ and $h$ parameters were found using least squares method in Matlab.

Comparison and consistent fitting of measured data and force model using equation 2 is shown in Figure 6.

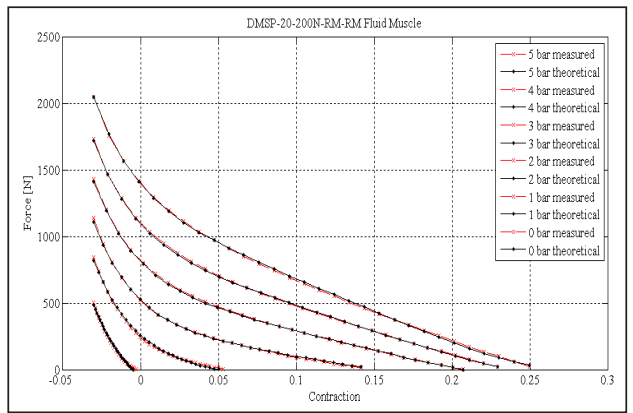

Figure 6. Comparison of measured data and our force model

Numbers have investigated the precise position control of pneumatic muscles during the past several years, too ${ }^{2,4,7,8,9}$. Most of them dealt with the control of single or antagonistic pneumatic muscles. The positioning of PAMs requires accurate determination of the dynamic model of pneumatic actuators. With the help our test-bed the hysteresis can be accurately predicted. Chou and Hannaford in ${ }^{4}$ report hysteresis to be substantially due to Coulomb friction, which is caused by the contact between the bladder and the shell, between the braided threads and each other, and the shape changing of the bladder. An experiment was made to illustrate the hysteresis (Figure 7).

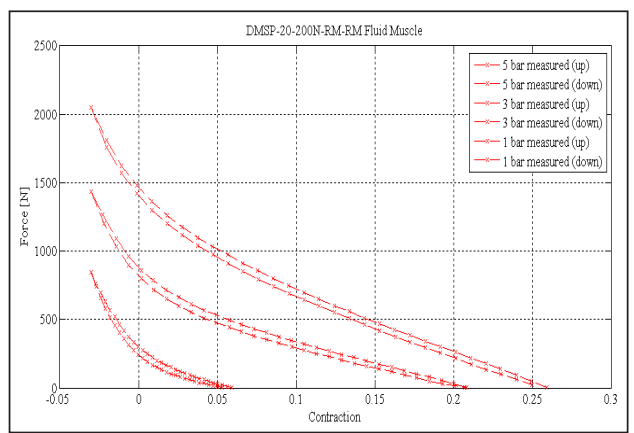

Figure 7. Hysteresis in the tension-length (contraction) cycle

To prove versatility of equation 2, another comparison was done between the measured data and force model. The accurate fitting is demonstrated in Figure 8.

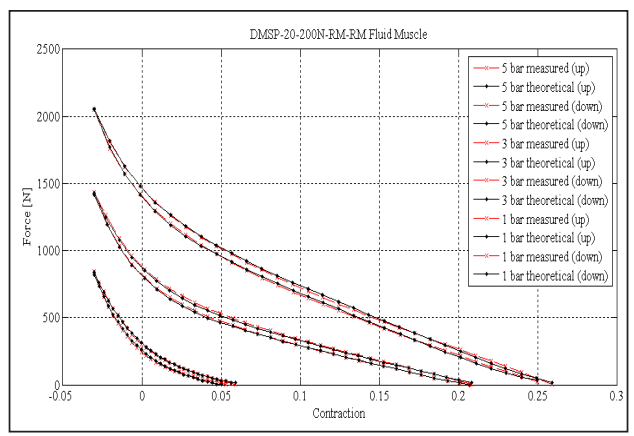

Figure 8. Approximation of hysteresis loop

Beside the traditional linear controllers several modern control methods with adaptive controller, fuzzy controller, neural network controller, sliding-mode controller among others have been developed. On the basis of slidingmode control, the next probe was a positioning with high-speed ON/OFF solenoid valves. The time functions of the position and control signal are shown in Figure 9. 


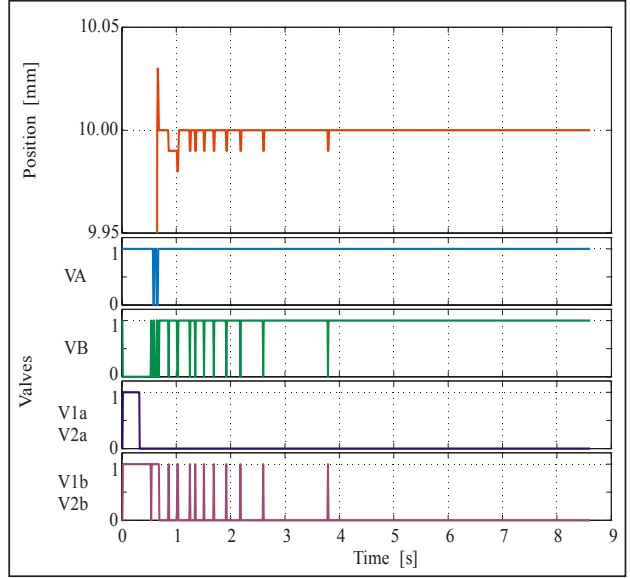

Figure 9. The time functions of the position and control signal (in LabVIEW environment)

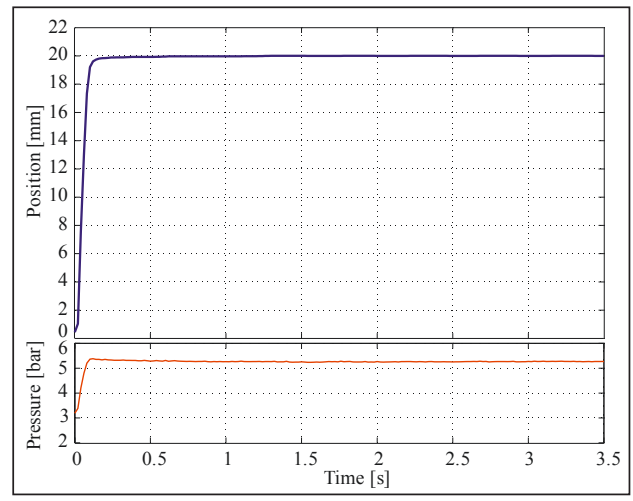

Figure 10. The time functions of the position and pressure (with PLC)

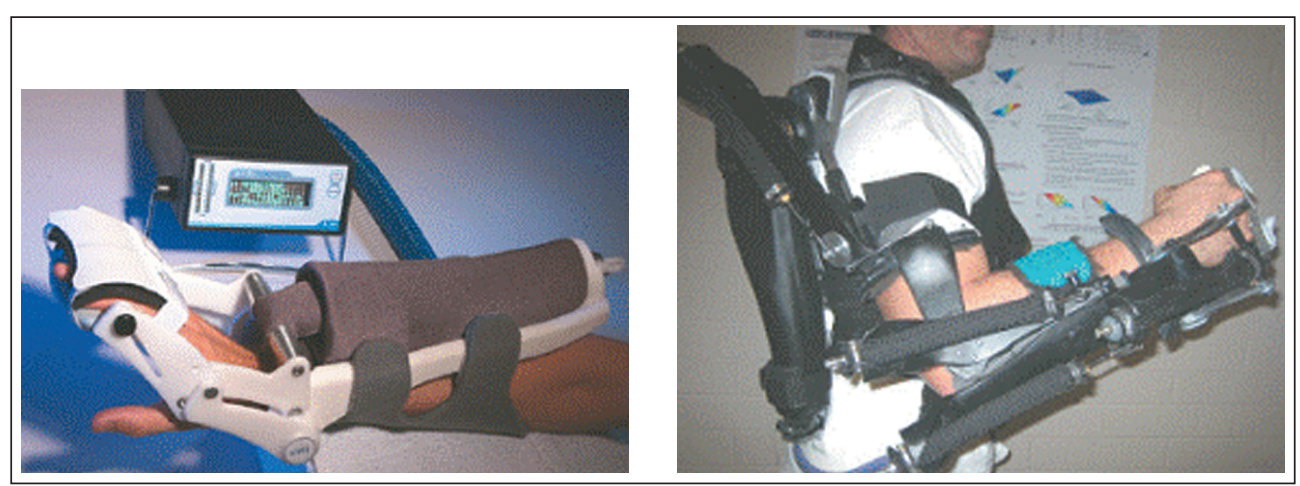

Figure 12. Mentor ${ }^{\mathrm{TM}}$ hand therapy device $\left(\mathrm{left}^{10}\right)$ and RUPERT IV upper extremity therapeutic device (right ${ }^{11}$ )
We repeated the previous experiment with PLC type Allen-Bradley CompactLogix L23E. Figure 10 and Figure 11 show the results.

Several therapy devices have been developed, but the typical robotic systems tend to be expensive and complex for clinical use and impossible for home use. Even, generally, these devices are built with electric motors and the movements are far from natural. The pneumatic artificial muscles are possible to be the actuators for these devices because of its soft motion and cheap and these devices can be used by patients at their own homes. Therapeutic devices provide training of reaching and feeding motions (Figure 12, 13).

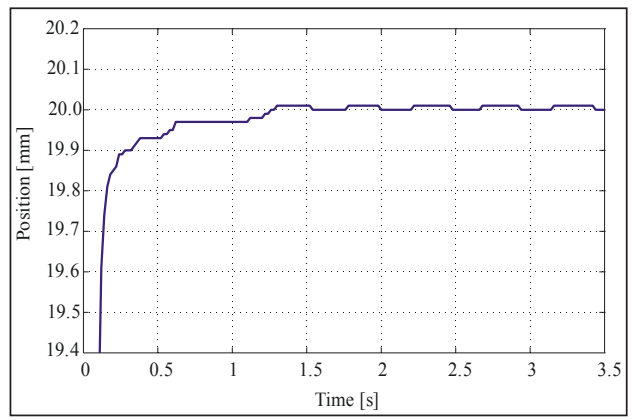

Figure 11. The time function of the position (with PLC, enlarged) 


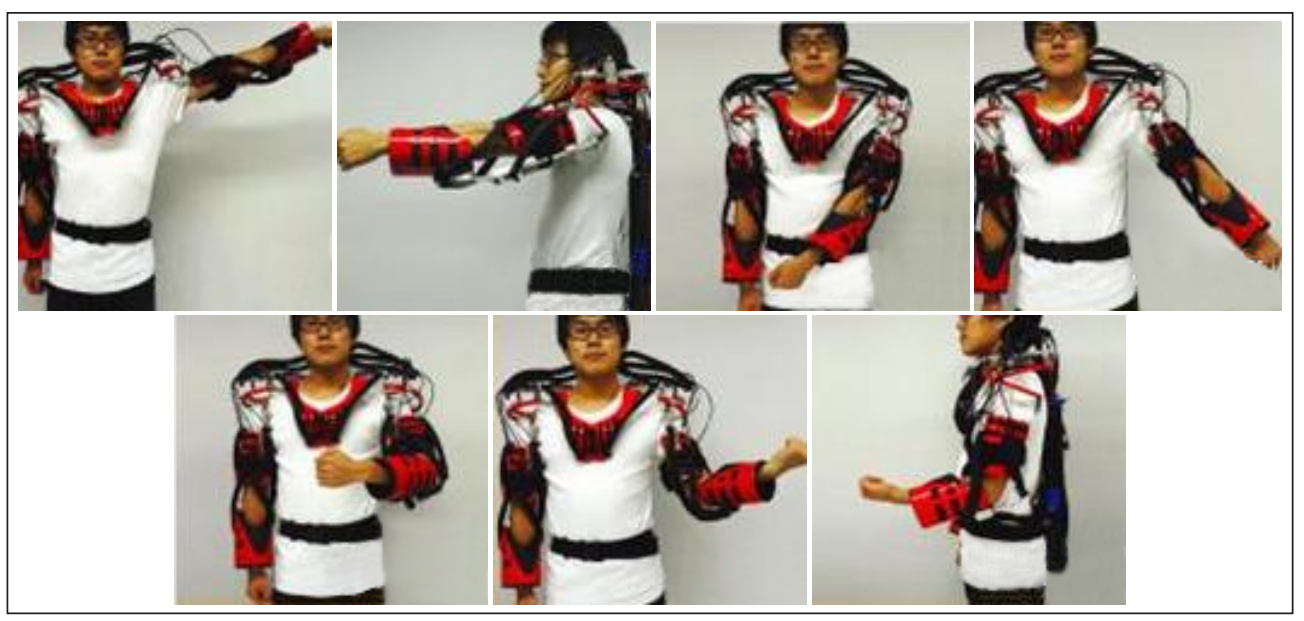

Figure 13. Muscle Suit ${ }^{12}$

Different prosthetic arms actuated by PAMs are shown in Figure 14. These were inspired by the human arm muscular system.

\section{Conclusions and future work}

The pneumatic artificial muscle is a pneumatic device characterized by its high level of functional analogy with human skeletal mus- cle. In this paper we have presented our testbed. With the help of it several static and dynamic investigations and control methods can be carried out. Some experimental results and rehabilitation devices and prosthetic arms using pneumatic artificial muscles have been shown, too. Our goal and future work is to construct a prosthetic arm with PAMs, because these muscles seem a better choice than present day electric or other drives.

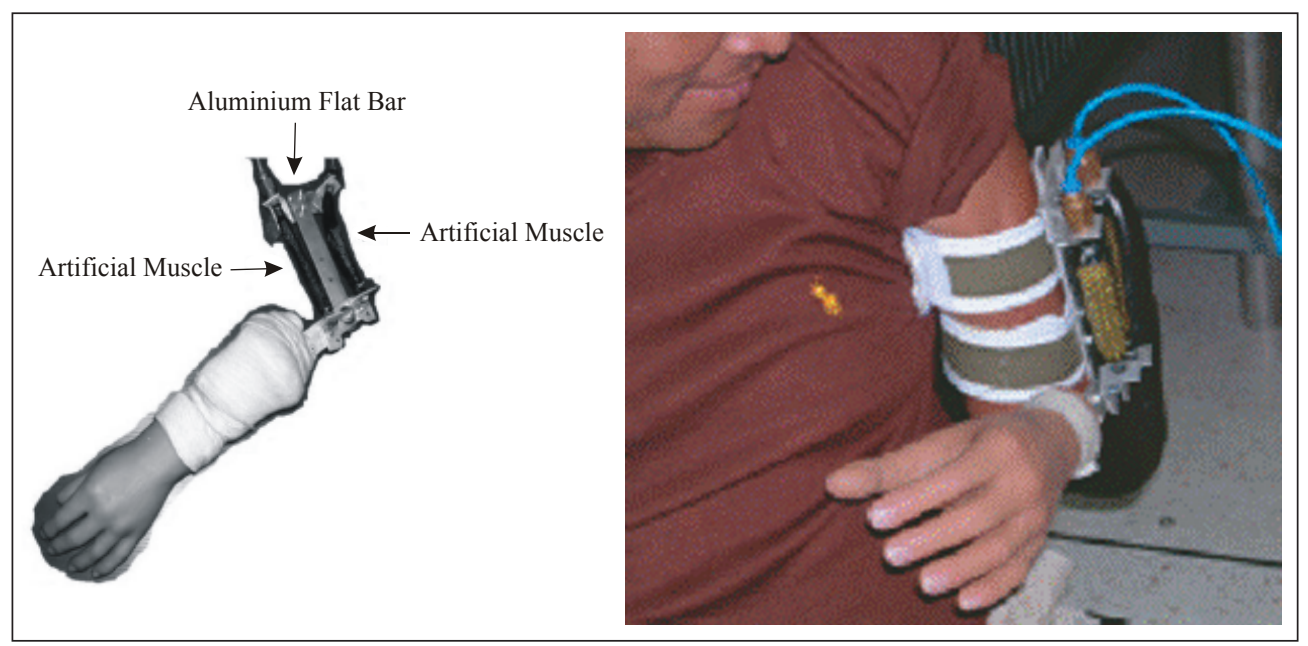

Figure 14. Artificial limbs with PAMs ${ }^{13,14}$ 


\section{REFERENCES}

1. Daerden F, Lefeber D. Pneumatic artificial muscles: actuator for robotics and automation. European Journal of Mechanical and Environmental Engineering 2002;47:10-21.

2. Situm Z, Herceg Z. Design and control of a manipulator arm driven by pneumatic muscle actuators. $16^{\text {th }}$ Mediterranean Conference on Control and Automation; 2008 Jun 25-27; Ajaccio, 2008;926-31.

3. Daerden F. Conception and realization of pleated artificial muscles and their use as compliant actuation elements. PhD Dissertation, Vrije Universiteit Brussel, Faculteit Toegepaste Wetenschappen Vakgroep Werktuigkunde 1999;9.

4. Chou CP, Hannaford B. Measurement and modelling of McKibben pneumatic artificial muscles. IEEE Transactions on Robotics and Automation 1996;12(1):90-102.

5. Versluys $R$, Naudet J, Vanderborght B, Lenaerts $G$, Lefeber $D$. Intelligent prosthesis actuated by pleated pneumatic artificial muscles. Proceeding on the $9^{\text {th }}$ International Conference on Climbing and Walking Robots 2006 Sept 12-14, Brussels; 2006;1-9.

6. Sárosi J, Gyeviki J, Szabó G, SzendrőP. Laboratory investigations of fluid muscles. Annals of Faculty of Engineering Hunedoara, International Journal of Engineering 2010;8(1):137-42.

7. Tondu B, Lopez P. Modelling and control of McKibben artificial muscle robot actuator. IEEE Control System Magazine 2000;20:15-38.
8. Caldwell DG, Medrano-Cerda GA, Goodwin M. Control of Pneumatic Muscle Actuator. IEEE Control Systems Magazine 1995 Feb: 40-8.

9. Lilly JH, Yang L. Sliding-mode tracking for pneumatic muscle actuators in opposing pair configuration. IEEE Transaction on Control System Technology 2005;13(4).

10. Koeneman EJ, Schultz RS, WolfSL, Herring DE, Koeneman JB. A pneumatic muscle hand therapy device. Proceedings of the 26th Annual International Conference of the IEEE EMBS; 2004 Sept 14; San Francisco, 2004:2711-13.

11. Balasubramanian S, Wei R, Perez M, Shepard B, Koeneman E, Koeneman J, He J. RUPERT. An exoskeleton robot for assisting rehabilitation of arm functions. Virtual Rehabilitation 2008; 2008 Aug 25-27, Vancouver, 2008;163-7.

12. Kobayashi H, Hiramatsu K. Development of muscle suit for upper limb. Proceedings of the 2004 IEEE, International Conference on Robotics and Automation 2004 Apr 26-May 1; Tokyo, 2004:2480-5.

13. Wongsiri S, Laksanacharoen S. Design and construction of an artificial limb driven by artificial muscles for amputees. International Conference on Energy and the Environment; 2003 Dec 1112; Songkla, 2003;4.

14. Laksanacharoen S. Artificial muscle contsruction using natural rubber latex in Thailand. The $3^{\text {rd }}$ Thailand and Material Science and Technology Conference; 2004 Aug 10-11; Bangkok, 2004;3.

\author{
József Sárosi \\ Department of Technical and Process Engineering \\ Faculty of Engineering \\ University of Szeged \\ H-6724 Szeged, Mars tér 7. \\ Tel.: (+36) 62 546-021
}

